\title{
Protein kinase CK2 is a critical regulator of epithelial homeostasis in chronic intestinal inflammation
}

\author{
S Koch ${ }^{1}$, CT Capaldo ${ }^{1}$, RS Hilgarth ${ }^{1}$, B Fournier ${ }^{1}$, CA Parkos ${ }^{1}$ and A Nusrat ${ }^{1}$
}

The molecular mechanisms that restore intestinal epithelial homeostasis during colitis are incompletely understood. Here, we report that during intestinal inflammation, multiple inflammatory cytokines promote the activity of a master regulator of cell proliferation and apoptosis, serine/threonine kinase CK2. Enhanced mucosal CK2 protein expression and activity were observed in animal models of chronic colitis, particularly within intestinal epithelial cells (IECs). The in vitro treatment of intestinal epithelial cell lines with cytokines resulted in increased CK2 expression and nuclear translocation of its catalytic $\alpha$ subunit. Similarly, nuclear translocation of CK2 $\alpha$ was a prominent feature observed in colonic crypts from individuals with ulcerative colitis and Crohn's disease. Further in vitro studies revealed that CK2 activity promotes epithelial restitution, and protects normal IECs from cytokine-induced apoptosis. These observations identify CK2 as a key regulator of homeostatic properties of the intestinal epithelium that serves to promote wound healing, in part through inhibition of apoptosis under conditions of inflammation.

\section{INTRODUCTION}

Crypt epithelial homeostasis in the intestine is maintained through the tight regulation of intestinal epithelial cell (IEC) proliferation, migration, and luminal shedding. In disorders such as the chronic inflammatory bowel diseases (IBD), ulcerative colitis and Crohn's disease, persistently elevated mucosal cytokine expression and leukocyte infiltration lead to IEC apoptosis and subsequent crypt hyperproliferation, which contribute to widespread tissue ulceration and epithelial hyperplasia. ${ }^{1}$ Although several conserved signaling pathways have been proposed to regulate tissue homeostasis during colitis, it remains incompletely understood how inflammatory mediators affect epithelial cell apoptosis and proliferation in chronic inflammation.

We recently reported that inflammatory cytokines, particularly interferon- $\gamma($ IFN- $\gamma)$ and tumor necrosis factor- $\alpha$, sequentially modulate IEC proliferation and apoptosis by transactivation of the major crypt homeostatic Wnt/ $\beta$-catenin and $\mathrm{Akt} / \beta$-catenin signaling pathways. ${ }^{2}$ In subsequent experiments aimed at identifying additional Wnt signaling-related molecules that may promote IEC survival during colitis, we observed an increase in serine/threonine kinase CK2 expression and activity in chronically inflamed intestinal mucosa. This prompted us to investigate the role of $\mathrm{CK} 2$ in colitis, because the kinase is known to be crucially involved in the regulation of various essential cellular processes, including cell survival, cell cycle control, and proliferation. ${ }^{3,4}$ In addition and of importance for this study, CK2 has been shown to promote mitogenic Wnt/ $\beta$-catenin signaling, by stabilizing disheveled and $\beta$-catenin proteins. ${ }^{5}$ Furthermore, previous studies have shown that CK2 inhibits epithelial cell apoptosis, among others by attenuating the activation of caspases 9 and 3.,7 Protein expression and activity of CK2 (consisting of two catalytic $\alpha$ and/or $\alpha^{\prime}$ subunits, and two regulatory $\beta$ subunits) are tightly regulated in normal tissues; however, increased CK2 levels are observed in virtually all cancers, ${ }^{8}$ including colorectal carcinomas. ${ }^{9,10}$ In contrast, very little is known about the regulation of CK2 under inflammatory conditions, and its contribution to the control of epithelial homeostasis remains to be determined. To our knowledge, only one prior report has shown increased expression of the CK2 $\alpha$ subunit in glomerulonephritis, and inhibition of CK2 activity attenuated disease progression in this study. ${ }^{11}$

We now report that inflammatory cytokines enhance CK2 $\alpha$ expression in IECs, and that CK2 expression and activity are

\footnotetext{
${ }^{1}$ Epithelial Pathobiology and Mucosal Inflammation Research Unit, Department of Pathology and Laboratory Medicine, Emory University, Atlanta, Georgia, USA. Correspondence: A Nusrat (anusrat@emory.edu) or S Koch (Stefan.koch@dkfz-heidelberg.de)

Received 25 October 2011; accepted 29 May 2012; published online 4 July 2012. doi:10.1038/mi.2012.57
} 
increased in chronic colitis. In addition, we show that cytokines induce rapid nuclear translocation of CK2 $\alpha$ in vitro and in vivo, which is thought to be a major regulatory mechanism of CK2 activity. ${ }^{12}$ Finally, we provide evidence that CK2 promotes the proliferation, survival, and migration of normal IECs, and that it protects epithelial cells from cytokine-induced apoptosis. Taken together, these observations suggest that CK2 is a critical regulator of crypt epithelial homeostasis in chronic intestinal inflammation.

\section{RESULTS \\ CK2 $\alpha$ expression and activity are altered in intestinal inflammation}

While investigating key signaling molecules likely to be involved in the regulation of $\beta$-catenin signaling and epithelial cell survival during intestinal inflammation, we observed increased expression of CK2 $\alpha$ in chronic dextran sulfate sodium (DSS)induced colitis. We subsequently determined the temporal regulation of CK $2 \alpha$ expression using animal models of acute colitis, recovery from acute intestinal inflammation, and chronic colitis (Figure 1a,b). Using two antibodies specific for CK2 $\alpha$, we found that protein expression in total mucosal lysates was dramatically reduced in acute colitis, but increased to approximately twice the level of healthy controls in chronic colitis. This observation was reflected in altered phosphorylation of kinase substrate $\mathrm{Cdc} 37$ at $\mathrm{S} 13$, which serves as a robust surrogate in vivo marker of CK2 activity. ${ }^{13}$ Initial immunolocalization studies indicated that within the intestine, CK2 $\alpha$ is strongly expressed in IECs. Thus, to confirm increased protein expression in epithelial cells, and to validate our findings using a separate disease model, we isolated IECs from mice with chronic DSS colitis as well as animals with chronic bacterial colitis induced by the enteropathogen Salmonella enterica, and assessed CK2 $\alpha$ and $\alpha^{\prime}$ expression (Figure 1c). CK2 $\alpha$ protein expression was significantly increased in both disease models. Additionally, we observed a moderate increase in CK2 $\alpha^{\prime}$ expression in chronic DSS colitis. To address whether these changes were associated with altered mRNA expression, we determined the relative amount of $\operatorname{csn} k 2 a 1$ (CK2 $\alpha), \operatorname{csn} 22 a 2\left(\mathrm{CK} 2 \alpha^{\prime}\right)$, and $\operatorname{csn} k 2 b$ $(\mathrm{CK} 2 \beta)$ transcripts in these samples (Figure 1d). Expression of all three mRNAs was unchanged or lower than in controls, consistent with the observation that CK2 gene products negatively regulate their own mRNA transcription. ${ }^{14}$ Finally, because elevated mucosal cytokine production is a characteristic feature of IBD, we investigated if inflammatory mediators could enhance CK2 expression in normal IEC-6 IECs, which exhibit physiologically low CK $2 \alpha$ expression and activity compared with commonly used cancer cell lines, including SW480 and SK CO-15 (Supplementary Figure S1A online). Preliminary experiments indicated that multiple pro- and anti-inflammatory cytokines increased CK2 $\alpha$ expression after extended exposure (7 days), that this induction occurred at pathophysiologically relevant cytokine concentrations, ${ }^{2,15}$ and that no apparent increase was seen after short treatment (1-3 days; Supplementary Figure S1B-D online). In particular, interleukin (IL)- $1 \beta$ and IL- 6 significantly increased CK $2 \alpha$ expression after treatment for 7 days (Figure 1e), suggesting that inflammatory cytokines may regulate CK2 activity during chronic inflammation.

\section{CK2 $\alpha$ translocates to the nucleus during intestinal inflammation}

Nuclear translocation of CK2 is thought to increase its cytoprotective activity, ${ }^{12}$ and strong nuclear localization of CK $2 \alpha$ has been demonstrated in colorectal cancers. ${ }^{9}$ Having shown that intestinal inflammation enhances CK2 $\alpha$ protein expression, we thus asked whether chronic colitis is also associated with nuclear translocation of this protein. Immunostaining of mouse tissue samples mainly showed diffuse cytoplasmic localization of CK2 $\alpha$ in the IECs of healthy mice (not shown). In contrast, we observed considerable nuclear accumulation of CK2 $\alpha$ in the IECs of animals with chronic inflammation, especially at the margins of mucosal ulcers (Figure 2a, and Supplementary Figure S2A online). Fidelity of the immunohistochemical staining protocol was confirmed by staining for proliferating cell nuclear antigen and $\beta$-catenin, which correctly localized to crypt base nuclei and epithelial plasma membranes, respectively (Supplementary Figure S2B online). A similar staining pattern as for CK2 $\alpha$ was observed for Cdc37 pS13, indicative of increased nuclear CK2 activity in regenerating and chronically inflamed colonic epithelium (Supplementary Figure S2C online). An analysis of CK2 $\alpha$ nuclear vs. cytoplasmic staining as a function of distance from ulcer revealed an exponential reduction in nuclear localization with increasing distance $\left(R^{2}=0.935\right.$; Figure 2b). We next investigated whether nuclear translocation of CK $2 \alpha$ also occurs in human tissue. Immunolocalization studies on noninflamed human colonic samples revealed that CK $2 \alpha$ is primarily found in the cytoplasm of IECs in the proliferative crypt base region (Figure 3a). Additional limited nuclear staining was seen in IECs close to the luminal surface. In contrast, we observed a considerable increase in CK2 $\alpha$ nuclear signal intensity in acutely inflamed ulcerative colitis and Crohn's disease samples, which was remarkably similar to colorectal cancer tissues that served as positive controls (Figure $3 \mathbf{b}$ ). ${ }^{9}$ Quantification of nuclear CK2 $\alpha$ signal intensity at the crypt base showed a highly significant difference compared with non-IBD controls, indicative of increased CK2 $\alpha$ nuclear translocation and/or protein expression in IBD (Figure 3c). To address whether CK2 $\alpha$ nuclear localization reflects the activity of inflammation, we localized this protein in matched quiescent and inflamed tissue samples from patients with IBD (2 ulcerative colitis and 1 Crohn's disease; Figure 3d). CK2 $\alpha$ nuclear signal intensity was moderately but highly significantly elevated in noninflamed areas compared with healthy controls $(1.3 \pm 0.1$-fold; $P<0.001)$. A further significant increase was seen in acutely inflamed samples $(2.0 \pm 0.2$-fold; $P<0.001)$. In addition, whereas nuclear staining in surface IECs was limited in noninflamed tissue, we observed robust staining in $\sim 10-20 \%$ of surface cells in IBD samples (Figure 3e). Because CK2 $\alpha$ expression was increased in IECs treated with inflammatory cytokines, we investigated whether these cytokines also promoted nuclear translocation. Indeed, IL- $1 \beta$, IL- 6 , IFN- $\gamma$, and tumor necrosis factor- $\alpha$ 


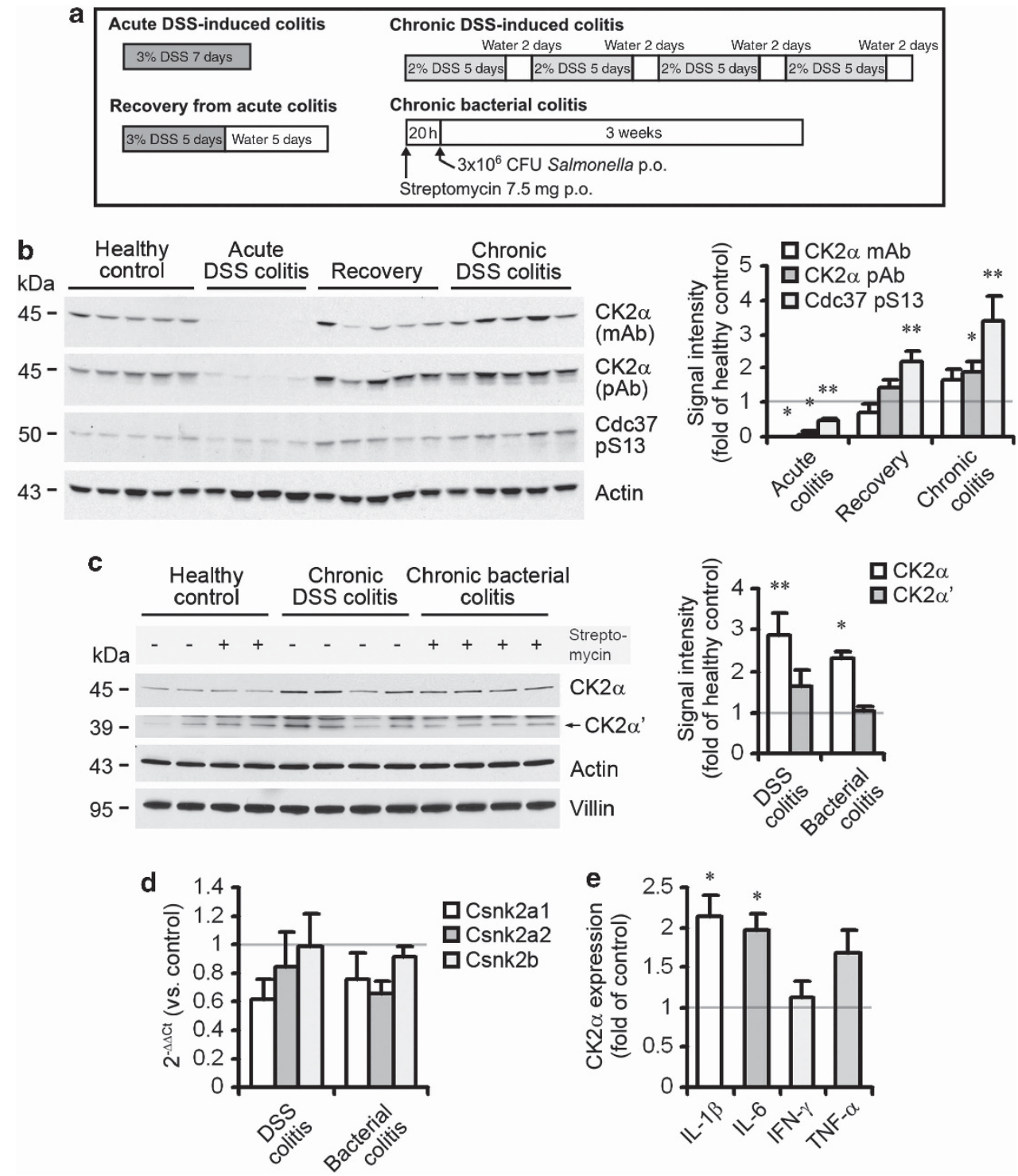

Figure 1 CK2 protein expression and activity are increased in chronic intestinal inflammation. (a) Acute and chronic colitis was induced as depicted in the schematic. (b) Total mucosal lysates were blotted for CK2 $\alpha$ using a monoclonal (mAb) and polyclonal (pAb) antibody, as well as CK2 substrate Cdc37 pS13. Densitometric analysis of the results is shown on the right. (c) Intestinal epithelial cells (IECs) were isolated from mice with chronic colitis and controls, and probed for CK2 $\alpha$ and CK2 $\alpha^{\prime}$ expression. (d) RNA was extracted from isolated IECs, and relative expression of CK2-encoding genes was determined by real-time reverse-transcriptase-PCR (RT-PCR); $n=3$ mice per group. (e) IEC- 6 cells were treated with the indicated cytokines $\left(10 \mathrm{ng} \mathrm{ml}^{-1}\right)$ for 7 days, and CK2 $\alpha$ expression was then determined by immunoblotting; $n=4$ independent experiments. DSS, dextran sulfate sodium; IFN- $\gamma$, interferon- $\gamma$; IL, interleukin; TNF- $\alpha$, tumor necrosis factor- $\alpha .{ }^{*} P<0.01 ;{ }^{* *} P<0.05$.

significantly enhanced CK2 $\alpha$ nuclear translocation in IEC-6 cells after treatment for $24 \mathrm{~h}$ (Figure 4a). Importantly, CK2 $\alpha$ protein expression was unchanged at this time point (Supplementary Figure S1D,E online), indicating that cytokines facilitate translocation of CK2 from the cytoplasm to the nucleus before affecting protein levels. Finally, to determine whether this was a transient effect, cytokines were washed out after $24 \mathrm{~h}$, and cells were allowed to recover in normal media for an additional $24 \mathrm{~h}$. Cytokine withdrawal restored CK2 $\alpha$ nuclear/ cytoplasmic distribution to baseline levels (Figure $4 b$ ), suggesting that CK2 nuclear localization is an immediate but reversible stress response.

\section{CK2 protects IECs from caspase-mediated apoptosis and promotes epithelial wound closure}

Because we observed increased CK2 expression and activity in chronic colitis, we next sought to determine the role of CK2 in IEC apoptosis and proliferation, particularly in noncancer epithelial cells. Caspase-9 is a major initiator caspase involved in IEC apoptosis, which has been shown to be stabilized by CK2. ${ }^{6}$ Accordingly, we observed marked cleavage of caspase- 9 in IEC- 6 cells treated with the specific CK2 inhibitors 4,5,6, 7-tetrabromobenzotriazole (TBB), tetrabromocinnamic acid (TBCA), and 3-methyl-1,6,8-trihydroxyanthraquinone (emodin), especially at concentrations of $10-25 \mu \mathrm{M}$ (Figure 5a, 
a
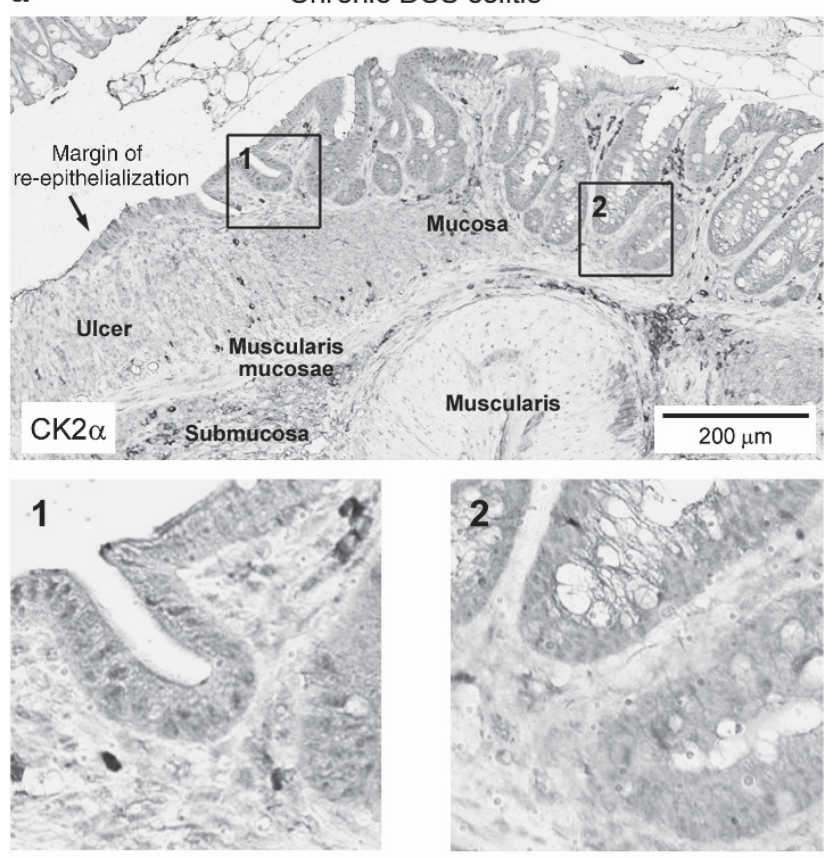

b

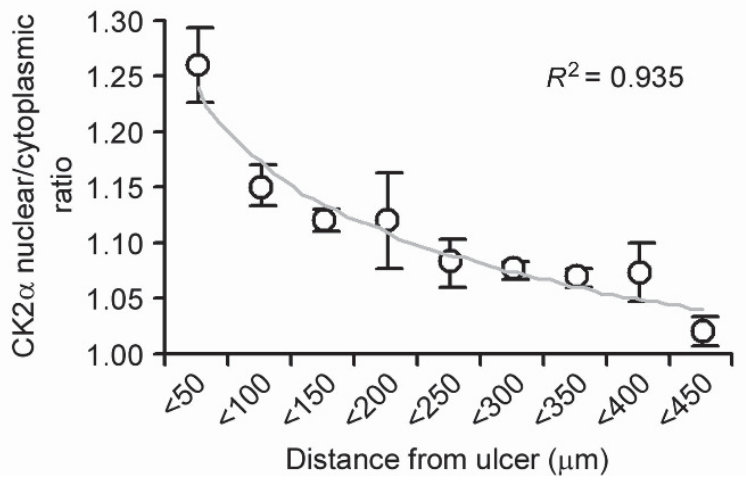

Figure 2 Intestinal inflammation is associated with nuclear translocation of CK2 $\alpha$. (a) CK2 $\alpha$ expression and subcellular localization in chronically inflamed mouse colon were determined by immunohistochemistry. Magnified insets show crypts immediately adjacent to (1) and somewhat removed from (2) a mucosal ulcer. (b) Analysis of CK2 $\alpha$ nuclear/cytoplasmic ratio in the intestinal epithelial cells (IECs) of mice with chronic dextran sulfate sodium (DSS) colitis. Results are pooled from six ulcer margins in three mice. The trendline indicates an exponential decrease with increasing distance from the ulcer.

and Supplementary Figure S3A online). At these inhibitor concentrations we did not observe inhibition of the potential off-target glycogen synthase kinase- $3 \beta,{ }^{16}$ indicating appreciable drug specificity (Supplementary Figure S3A online). Most consistent effects were seen with inhibitor concentrations of $25 \mu \mathrm{M}$, which were therefore used in most subsequent experiments; however, caspase-3-positive apoptotic cell clusters were observed after treatment with inhibitor concentrations as low as $6 \mu \mathrm{M}$ (Supplementary Figure S3B online). To confirm these observations and establish a specific role for the $\alpha$ subunit of CK2, we depleted CK2 $\alpha$ in highly transfectable SK-CO15 cells (Figure 5b). Loss of CK2 $\alpha$ induced substantial cleavage of caspase- 3 substrate poly(ADP-ribose) polymerase, thus confirming a critical role of CK2 $\alpha$ in IEC survival. In addition, apoptosis induced by IFN- $\gamma$ and tumor necrosis factor- $\alpha$ was further enhanced by CK $2 \alpha$ depletion. Similarly, partial depletion of CK2 $\alpha$ expression in SW480 IEC cancer cells sensitized the cells to camptothecin-induced caspase- 9 activation (Figure 5c). In agreement with these observations, treatment of IEC- 6 cells with a low dose of TBB, which by itself did not considerably affect IEC survival, promoted caspase- 3 activation in the presence of IFN $-\gamma$ and tumor necrosis factor- $\alpha$ (Supplementary Figure S3C online). These results suggest that increased CK2 activity protects IECs from cytokine-induced apoptosis in chronic inflammation. Consistent with this hypothesis, we found that in IBD tissue samples, apoptotic IECs at the luminal surface exhibited significantly lower CK2 $\alpha$ nuclear staining (Figure 5d). Because increased IEC apoptosis may attenuate epithelial wound closure, we next investigated the effect of CK2 inhibitors on epithelial restitution using an in vitro scratchwounding assay. Compared with dimethyl sulfoxide control, TBB dose-dependently inhibited IEC-6 migration (Figure 5e), which was statistically significant across multiple experiments at $10 \mu \mathrm{M}(P=0.01)$ and $25 \mu \mathrm{M}(P<0.01)$. Similar results were obtained with TBCA and emodin (Supplementary Figure S4A online). CK2 inhibitors did not affect lamellipodia dynamics or leading edge cell velocity, as indicated by live cell microscopy (Supplementary Figure S4B online). In contrast, immunostaining of IEC-6 cells revealed considerably increased caspase-9 activation along the wound edge of monolayers treated with TBB, TBCA, or emodin for $4 \mathrm{~h}$ (Figure 5f, and Supplementary Figure S4C online). Consistently, co-treatment of cells with caspase-9 inhibitor zLEHD-fmk enhanced epithelial restitution attenuated by TBB, which suggests that CK2 promotes epithelial wound healing at least in part by inhibiting caspase-mediated IEC apoptosis.

\section{CK2 regulates $\beta$-catenin activation and proliferation in IECs}

CK2 is a critical regulator of $\beta$-catenin signaling and cell proliferation; ${ }^{3,5}$ however, its function in IECs is incompletely understood and has largely been studied in cancer cell lines. We observed that enhanced expression of CK $2 \alpha$ and $\alpha^{\prime}$ in IEC- 6 cells treated with inflammatory cytokines is associated with an increase in $\beta$-catenin expression (Figure 6a), indicative of CK2-dependent, increased $\beta$-catenin protein stability. ${ }^{5}$ In addition, CK2 promoted Wnt $/ \beta$-catenin signaling in IECs, as determined using a $\beta$-catenin/T-cell factor (TCF) reporter construct (TOPflash) (Figure 6b). CK2 has been shown to promote $\beta$-catenin transcriptional activity in HEK 293T cells, by enhancing its transactivation through protein kinase Akt. ${ }^{17}$ In agreement with these findings, we observed that under low serum conditions, reduction of CK2 activity for $6 \mathrm{~h}$ using inhibitor TBB markedly decreased CK2-dependent phosphorylation of Akt at S129, and Akt-dependent phosphorylation of $\beta$-catenin at S552 (Figure 6c). Interestingly, this effect was not reversed by addition of insulin or epidermal growth factor (Figure $\mathbf{6 c}$, and data not shown), but could be inhibited by supplementation with $10 \%$ fetal bovine serum, suggesting that serum proteins may promote 

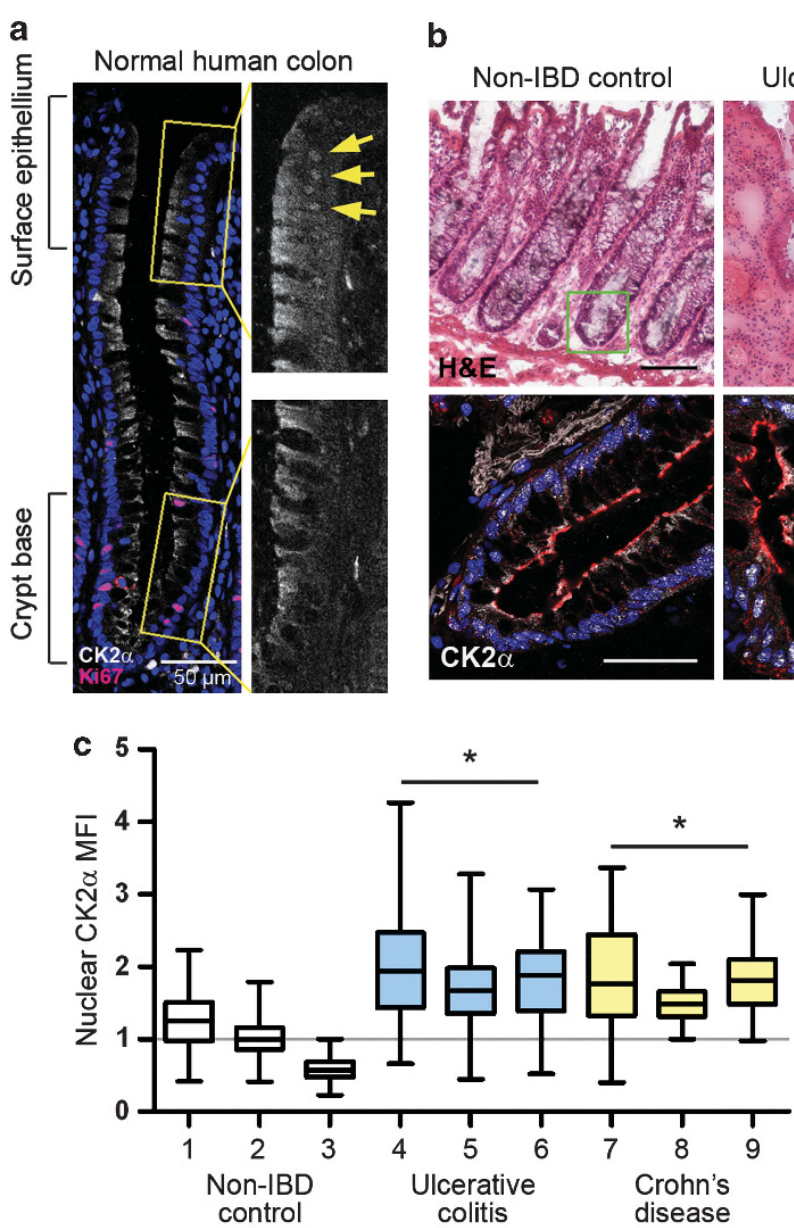

b
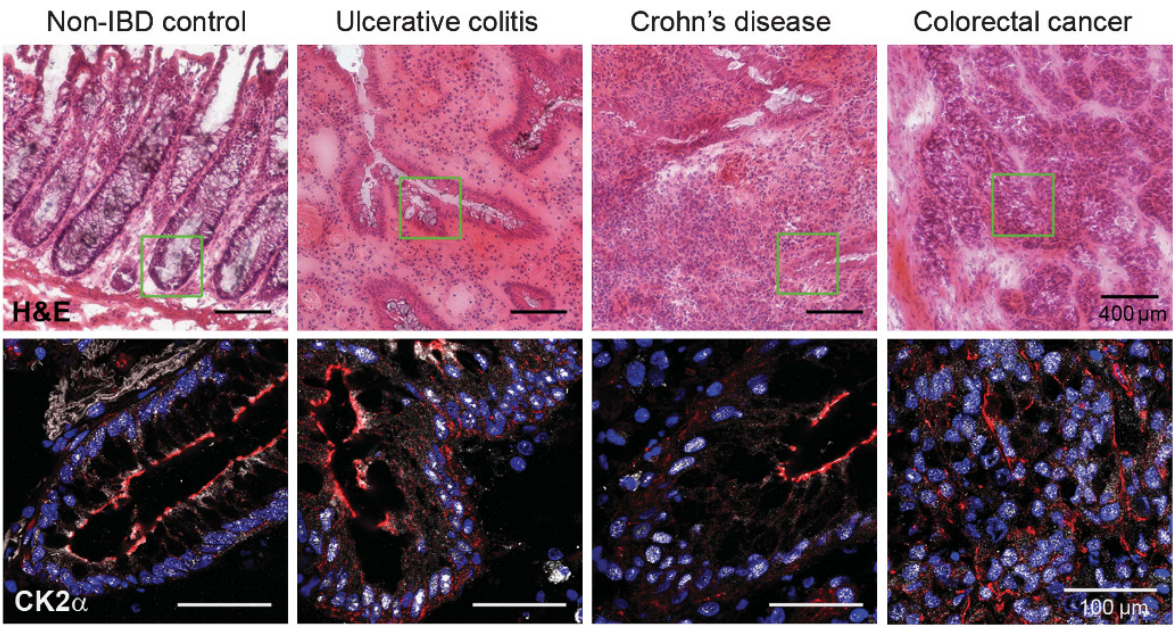

d
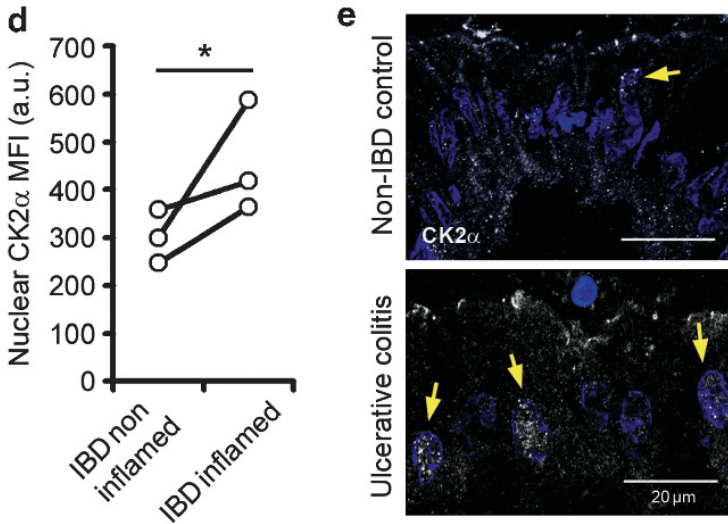

Figure 3 Nuclear localization of CK2 $\alpha$ is observed in human inflammatory bowel disease (IBD). (a) Representative CK2 $\alpha$ staining in normal human colon. The crypt base proliferative compartment is highlighted by Ki67 staining. Arrows show nuclear localization in intestinal epithelial cells (IECs) at the crypt-surface interface. (b) Hematoxylin and eosin (H\&E; upper panels) and CK2 $\alpha$ staining (lower panels; magnification of highlighted areas) of control and acutely inflamed IBD tissue. Colorectal cancer samples served as control. Slides were counterstained with phalloidin (red) to highlight the crypt lumen. (c) Quantification of nuclear CK2 $\alpha$ mean fluorescence intensity (MFI) in control and IBD tissues. Results were normalized to the average MFI of controls, and displayed as box-and-whisker plot with mean and quartiles \pm maximum/minimum; $n>50 \mathrm{IEC}$ per data point. 1,2 : histologically normal cancer margin; 3: benign polyp. (d) CK2 $\alpha$ nuclear MFI (arbitrary units (a.u.)) in matched quiescent and inflamed mucosal samples from patients with IBD; $n>100$ IECs per data point. (e) CK2 $\alpha$ staining in surface IECs. Arrows highlight strong nuclear $C K 2 \alpha$ signal. * $P<0.01$.

CK2 activity. Because $\beta$-catenin signaling is a major mitogenic stimulus in IEC, ${ }^{1}$ we next determined the effect of CK2 inhibition on epithelial cell proliferation. Treatment of IEC-6 cells with TBB for $6 \mathrm{~h}$ under serum-reduced conditions completely blocked proliferation, as determined by a lack of 5-ethynyl2 '-deoxyuridine (EdU) incorporation, which was accompanied by marked nuclear condensation indicative of IEC apoptosis (Figure 6d). This effect was largely rescued by addition of $10 \%$ fetal bovine serum. Conversely, overexpression of CK $2 \alpha$ in IEC- 6 cells increased baseline proliferation indicated by increased EdU staining (Supplementary Figure S4D,E online). However, CK2 $\alpha$ overexpression did not rescue cytokineinduced reduction in proliferation, potentially because of the modest increase in CK2 levels achieved in these experiments. Taken together, these results show that CK2 promotes $\beta$-catenin signaling and cell proliferation in IECs.

\section{DISCUSSION}

Protein kinase CK2 is critically involved in many homeostatic signaling events, and its expression and activity is increased in virtually all cancers, where it is thought to confer growth benefits and apoptosis resistance to cancer cells. ${ }^{3,4,8}$ However, the role of CK2 in inflammatory disorders is incompletely understood. We now report the novel observations that CK2 $\alpha$ expression and nuclear translocation are increased in chronic intestinal inflammation, that these effects are at least in part facilitated by inflammatory cytokines, and that CK2 activity is required for normal IEC homeostasis. Increased CK2 $\alpha$ expression and activity have previously been reported in the inflammatory kidney disease glomerulonephritis. ${ }^{11}$ Yamada et al. ${ }^{11}$ showed that progressive kidney inflammation is associated with enhanced CK2 $\alpha$ expression in rodent models of glomerulonephritis, as well as human nephritis samples. In good agreement with these 

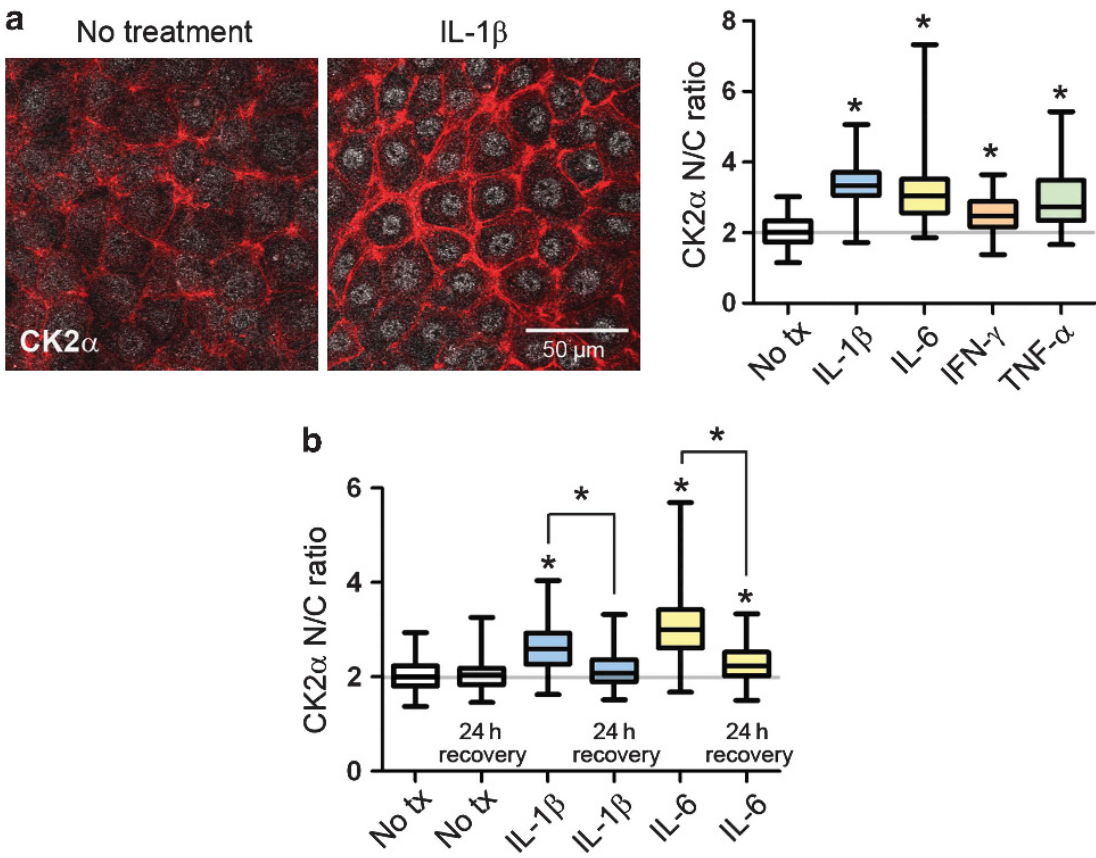

Figure 4 Inflammatory cytokines induce nuclear translocation of CK2 $\alpha$. (a) IEC-6 cells were treated with the indicated cytokines (10 ng ml ${ }^{-1}$ ) for 24h, and stained for CK2 $\alpha$ and filamentous actin (red). Increased actin stress fibers can be seen in cytokine-treated cells. Quantification of the nuclear/cytoplasmic (N/C) signal ratio is shown on the right. (b) IEC-6 cells were treated as indicated for $24 \mathrm{~h}$, or for $24 \mathrm{~h}$ followed by $24 \mathrm{~h}$ of media only. The graphs show one representative out of three independent experiments with $\geqslant 100$ cells per condition. Results in $\mathbf{a}$ and $\mathbf{b}$ are displayed as box-and-whisker plot with mean and quartiles \pm maximum/minimum. IEC, intestinal epithelial cell; IFN- $\gamma$, interferon- $\gamma$; IL, interleukin; TNF- $\alpha$, tumor necrosis factor- $\alpha .{ }^{*} P<0.01$.

observations, we found increased CK2 $\alpha$ protein expression in two mouse models of chronic intestinal inflammation, particularly in epithelial cells, and CK2 $\alpha$ nuclear translocation in inflamed mouse and human tissues. Importantly, this increase in protein levels and nuclear localization is very comparable to recent data on colorectal cancers, ${ }^{9,10}$ which suggest that our findings are highly relevant for the pathobiology of IBD and potentially colitis-associated cancers.

Interestingly, in contrast to the study by Yamada et al. ${ }^{11}$ we did not observe concomitantly increased mRNA expression of CK2encoding genes. This apparent discrepancy was also observed in pancreatic cancers, ${ }^{18}$ and may be explained by the observation that CK2 negatively regulates its own gene transcription. ${ }^{14}$ Pyerin and Ackermann ${ }^{14}$ reported that the holoenzyme phosphorylates transcription factor AP-1, thereby inhibiting CK2 promoter binding, which suggests that a decrease in CK2 mRNA transcription may reflect increased kinase activity. Our data thus support the hypothesis that CK2 protein expression is mainly regulated through altered protein stability, rather than enhanced gene transcription. ${ }^{18,19}$ This hypothesis is also consistent with our observation that increased CK2 protein expression was not seen at early time points. However, the molecular mechanisms resulting in CK2 accumulation in chronic inflammation remain to be elucidated, and may differ between diseases.

Furthermore, we report that a panel of pro- and antiinflammatory cytokines increases CK $2 \alpha$ expression, promotes CK2 $\alpha$ nuclear translocation, and that these changes are reflected in enhanced CK2 $\alpha$ expression and nuclear localization in chronic intestinal inflammation in vivo. These observations are consistent with prior studies showing that IL- $1 \beta$, IL- 6 , and IFN- $\gamma$ enhance CK2 activity. ${ }^{20-22}$ The wide range of inflammatory mediators that increase CK2 expression suggests that protein expression or stability may be regulated through common stress response signaling pathways, which are shared by most cytokines. ${ }^{1}$ For example, promoter analysis of the CK2encoding genes revealed that the expression of either CK2 subunit is controlled by transcription factors Ets-1, Sp-1, and nuclear factor- $\mathrm{\kappa B},{ }^{19,23,24}$ all of which are activated by cytokines through mitogen-activated protein kinase, IкB kinase, and Jak signaling pathways. It is thus possible that during the transition from acute to chronic colitis, inflammatory cytokines promote CK2 gene transcription.

Our data show that CK2 is a critical regulator of intestinal epithelial homeostasis. Interestingly, CK $2 \alpha$ protein expression appears to be highly dynamic in the progression from acute to chronic DSS colitis, with greatly reduced protein levels during acute inflammation. In contrast, our results suggest that nuclear accumulation of this kinase is independent from changes in protein expression, and may be regulated by different mechanisms. In epithelial cancer cells, both enhanced expression and nuclear translocation of CK $2 \alpha$ have been shown to promote proliferation, inhibit apoptosis, and enhance cell migration. . $^{8,10}$ However, whereas these characteristics are clearly detrimental in cancers, as they may enhance tumor growth and spreading, they are essential for IEC survival and epithelial regeneration during intestinal inflammation. Indeed, our data show that CK2 


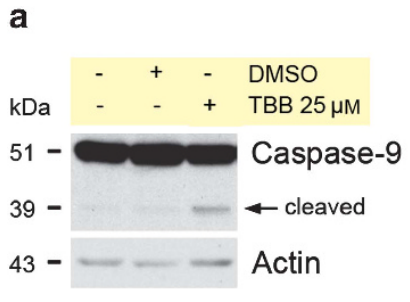

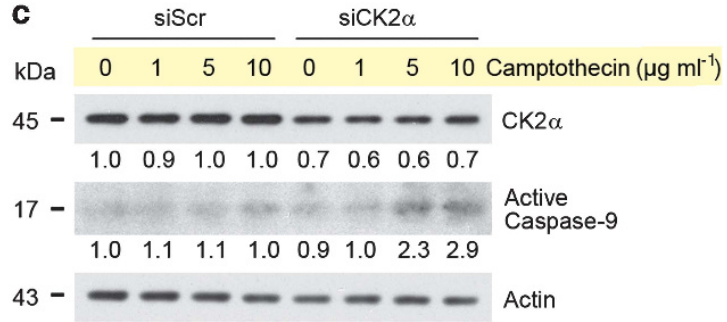

d

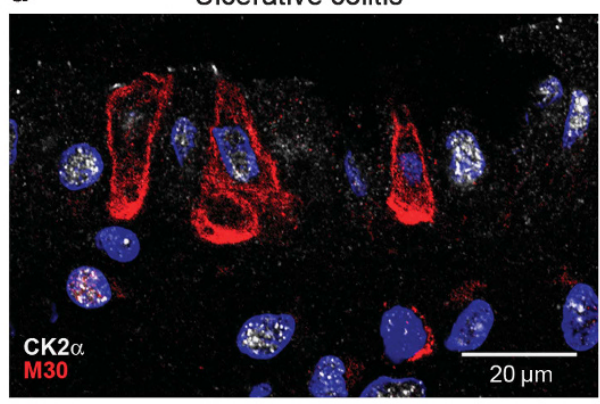

b

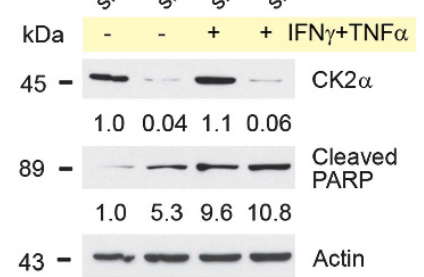

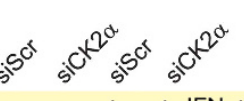

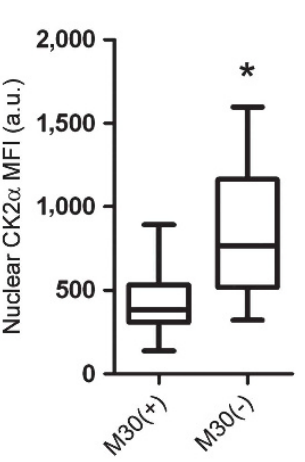

e

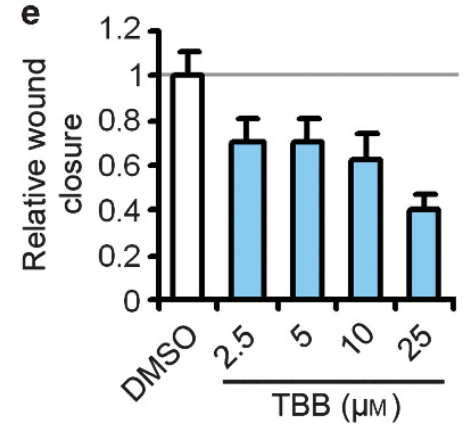

f

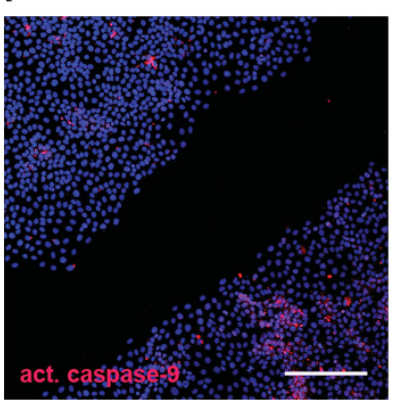

TBB $25 \mu \mathrm{m}$

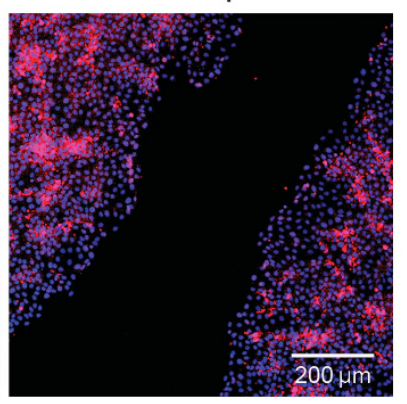

g

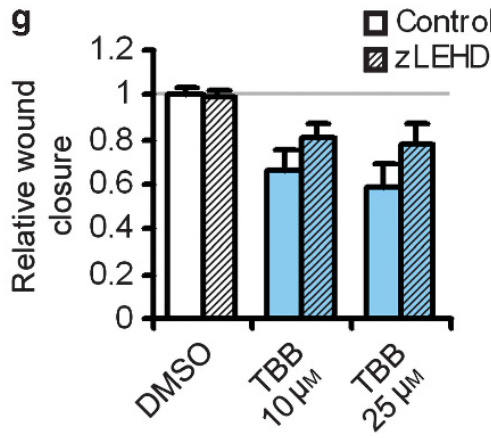

Figure 5 CK2 inhibits caspase-dependent intestinal epithelial cell (IEC) apoptosis and promotes cell migration. (a) IEC- 6 cells were treated as indicated for $8 \mathrm{~h}$, and probed for caspase-9 cleavage. DMSO, dimethyl sulfoxide; TBB, 4,5,6,7-tetrabromobenzotriazole. (b) CK2 $\alpha$ was depleted in SK-CO15 cells, and cells were then treated with cytokines (interferon (IFN): $50 \mathrm{Uml}^{-1}$; tumor necrosis factor (TNF): $20 \mathrm{ng} \mathrm{ml}^{-1}$ ) for $24 \mathrm{~h}$. Apoptosis induction was assessed by poly(ADP-ribose) polymerase (PARP) cleavage. Normalized signal intensity is indicated below the blots. (c) CK2 $\alpha$ was depleted in SW480 cells, and cells were treated with camptothecin for $4 \mathrm{~h}$. Normalized signal intensity is indicated below the blots. (d) Inflammatory bowel disease (IBD) tissue samples were stained for CK2 $\alpha$ and M30 (caspase-mediated cleavage of cytokeratin 18). A representative image of surface epithelium from an ulcerative colitis sample is shown on the left. The graph depicts nuclear CK2 $\alpha$ mean fluorescence intensity (MFI; arbitrary units (a.u.)) in normal and apoptotic IECs. Results are displayed as box-and-whisker plot with mean and quartiles \pm maximum/minimum. (e) IEC-6 cells were scratch-wounded and treated as indicated. Remaining wounds were measured after $8 \mathrm{~h}$. Results are representative of three independent experiments with six wells per condition. (f) Scratch-wounded IEC-6 cells were stained for cleaved caspase- 9 after $4 \mathrm{~h}$ of migration. (g) Cell migration was partially restored by addition of caspase-9 inhibitor ZLEHD-fmk $(5 \mu \mathrm{m})$. Wound closure was determined after $8 \mathrm{~h}$. Results are representative of two experiments with three wells per condition. ${ }^{\star} P<0.01$.

activity is critical for the proliferation and migration of normal IECs. In addition, we demonstrate that increased CK2 expression protects IECs from cytokine-induced apoptosis, which may be a protective response to chronically elevated mucosal cytokine concentrations. We thus hypothesize that increased CK2 expression and activity facilitate efficient epithelial regeneration, for example, in response to tissue ulceration. Consistent with this hypothesis, we observed that CK2 $\alpha$ nuclear localization was particularly evident in crypts adjacent to mucosal ulcers, which exhibit strongly increased cell proliferation. ${ }^{2,25}$ The role of epithelial cell apoptosis in the pathology of IBD remains somewhat controversial. Although increased IEC apoptosis is a common feature of both IBD and bacterial colitis, ${ }^{26}$ it is debatable whether apoptotic cell death contributes to the initiation of intestinal inflammation, or rather constitutes a secondary effect caused by accelerated crypt turnover. Nonetheless, it is feasible that enhanced CK2 activity accelerates mucosal wound healing by imposing a pro-proliferative, anti-apoptotic phenotype on epithelial crypts in the wound bed. In addition, the observation that nuclear CK2 $\alpha$ can be seen in surface epithelial cells in the normal mucosa suggests that CK2 may also be involved in the regulation of IEC shedding in the healthy intestine.

Conversely, persistently increased CK2 activity in chronic inflammation may have considerable negative consequences. For example, similar to the observations by Yamada et al., ${ }^{11}$ 

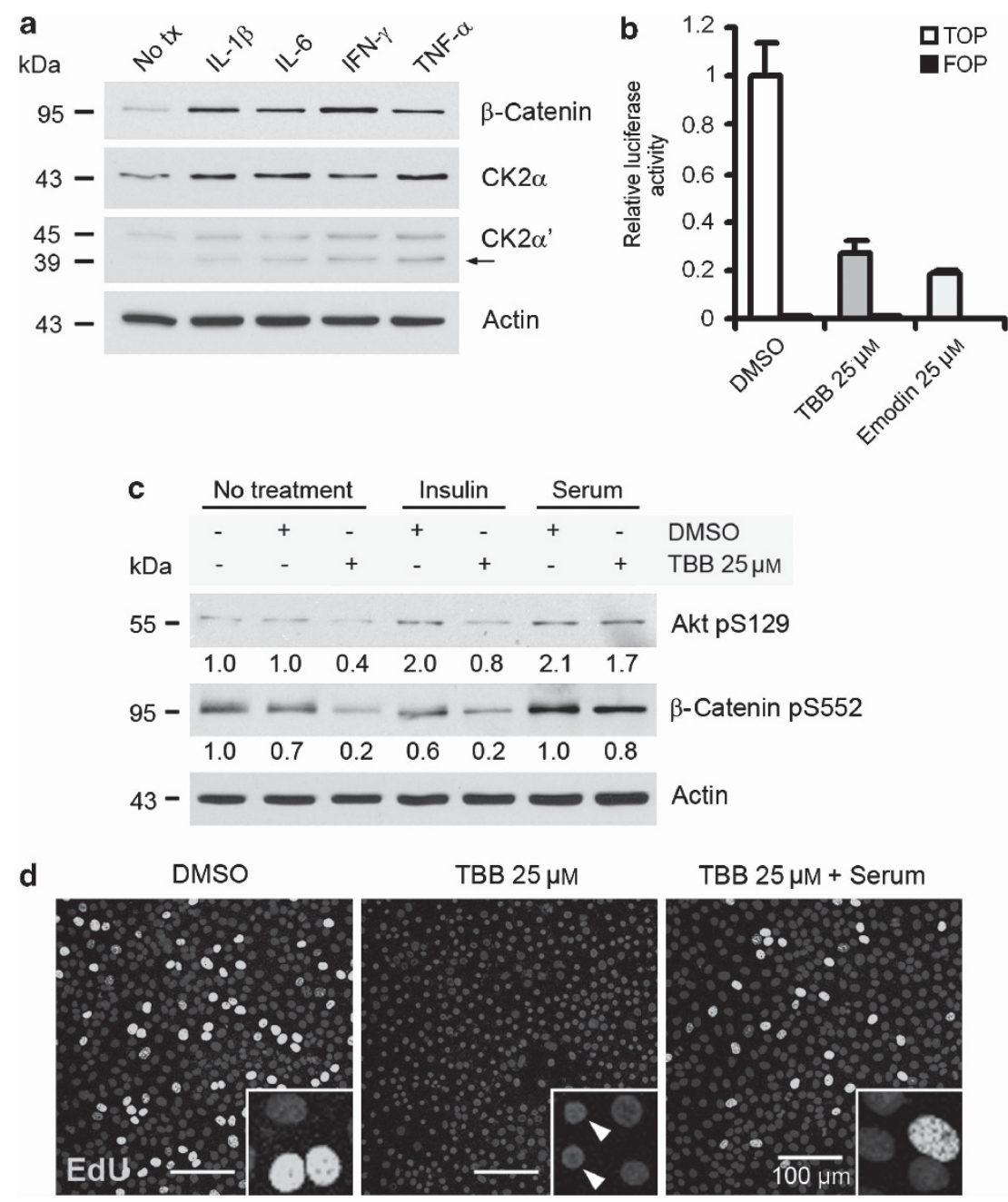

Figure 6 CK2 promotes $\beta$-catenin signaling and cell proliferation in intestinal epithelial cells (IECs). (a) IEC-6 cells were treated with the indicated cytokines $\left(10 \mathrm{ng} \mathrm{ml}^{-1}\right)$ for 7 days, and total cell lysates were probed for CK2 $\alpha$, CK2 $\alpha^{\prime}$, and $\beta$-catenin. (b) SK-CO15 cells were transfected with a $\beta$ catenin/TCF reporter construct (TOPflash/FOPflash) and treated as indicated for $8 \mathrm{~h}$. Experiments were performed in triplicate. (c) IEC- 6 cells were treated as indicated in serum-reduced media for $6 \mathrm{~h}$, and cell lysates were probed for active Akt and $\beta$-catenin. Normalized signal intensity is indicated below the blots. (d) IEC-6 cells were treated as indicated in serum-reduced media for $6 \mathrm{~h}$, and cell proliferation was determined by 5-ethynyl-2' deoxyuridine (EdU) incorporation for $30 \mathrm{~min}$. Arrowheads indicate nuclear condensation. DMSO, dimethyl sulfoxide; IFN- $\gamma$, interferon- $\gamma$; IL, interleukin; TBB, 4,5,6,7-tetrabromobenzotriazole; TNF- $\alpha$, tumor necrosis factor- $\alpha$.

increased CK2 activity in the intestinal lamina propria may enhance fibroblast proliferation and fibrosis, a common complication in Crohn's disease. Additionally, increased IEC apoptosis resistance could allow cancer-initiating cells to escape apoptotic clearance, and thereby contribute to colitis-associated carcinogenesis. Consistent with this hypothesis, prior studies using CK2 inhibitors in IEC cancer cell lines showed no or limited increase in epithelial cell death at inhibitor concentrations that cause complete loss of proliferation and gross apoptosis in normal IEC-6 cells. ${ }^{27-29}$ Finally, a recent report showed that CK2 enhances transepithelial permeability in IEC cancer cell lines. ${ }^{27}$ Increased CK2 activity may thus enhance the epithelial barrier defect seen in IBD.

In summary, we report that CK2 expression and activity are increased in chronic colitis, which is likely to be mediated by inflammatory cytokines. Based on these observations, we propose that modulation of CK2 activity during acute and chronic inflammation may provide a novel approach for enhancing epithelial restitution in IBD.

\section{METHODS}

Ethics statement. All animal experiments were approved by the Institutional Animal Care and Use Committee at Emory University, and performed according to the National Institutes of Health guidelines. Discarded human surgical specimens were used with approval from the institutional review board at Emory University Hospital.

Animal experiments. Induction of acute and chronic DSS colitis was performed as described previously, and depicted in Figure 1a. ${ }^{2,30}$ In brief, 2 or 3\% DSS wt/vol (lot 124156; USB Corporation, Cleveland, $\mathrm{OH}$ ) was dissolved in tap water and given ad libitum. Animals were monitored for changes in body weight, stool consistency, and presence of blood in the stool. Mice were killed after 7 days (acute colitis), 10 days (recovery phase), or 4 weeks (chronic colitis). Chronic bacterial 
colitis was induced as reported, ${ }^{31}$ with minor modifications. Briefly, mice were starved for $4 \mathrm{~h}$, and gavaged with $7.5 \mathrm{mg}$ streptomycin. After $20 \mathrm{~h}$, mice were gavaged with $3 \times 10^{6} \mathrm{CFU}$ attenuated, streptomycinresistant $S$. enterica serovar Typhimurium aro $^{-}$. Salmonella colonization was confirmed by plating of stool samples after $48 \mathrm{~h}$. Mice were killed after 3 weeks. IECs were isolated by incubation of intestines in Cell Recovery Solution (BD Biosciences, Franklin Lakes, NJ). Animal tissues were homogenized into RIPA lysis buffer $\left(150 \mathrm{mmoll}^{-1} \mathrm{NaCl}\right.$, $1 \%$ Nonidet P- $40,0.5 \%$ deoxycholic acid, $0.1 \%$ sodium dodecyl sulfate, and $50 \mathrm{mmoll}^{-1}$ Tris, $\mathrm{pH} 8.0$ ) with protease and phosphatase inhibitors (Sigma Aldrich, St Louis, MO) for immunoblotting, or Isol-RNA (5 PRIME, Gaithersburg, MD) for RNA extraction. For microscopy, pieces of colon were embedded in OCT (Sakura Finetek, Torrance, CA) and snap-frozen on dry ice, or in paraffin after fixation in formalin.

Reagents. The monoclonal CK2 $\alpha$ antibody used in most experiments was obtained from Millipore (Billerica, MA). Other antibodies were from Cell Signaling (Danvers, MA; CK2 $\alpha$ pAb, $\beta$-catenin pS552, caspase-9, cleaved poly(ADP-ribose) polymerase, histone $\mathrm{H} 3$, and glycogen synthase kinase-3 $\beta$ pS9), Assay Biotech (Sunnyvale, CA; Cdc37 pS13, Akt pS129, and cleaved caspase-9), R\&D Systems (Minneapolis, MN; cleaved caspase-3), Sigma Aldrich (actin, $\beta$-catenin, and villin), Aviva Systems Biology (San Diego, CA; CK2 $\alpha^{\prime}$ ), New England Biolabs (Ipswich, MA; SNAP), and Roche Diagnostics (Indianapolis, IN; M30). Small interfering RNA duplexes were from Sigma Aldrich, and transfected using Lipofectamine 2000 (Invitrogen, Carlsbad, CA). The EdU Click-IT kit was obtained from Invitrogen. CK2 inhibitors were purchased from Sigma Aldrich (TBB and emodin) and Calbiochem (Billerica, MA; TBCA), and dissolved in dimethyl sulfoxide. Equivalent concentrations of dimethyl sulfoxide were used in control conditions. The TOPflash/FOPflash assay was performed using the firefly/Renilla Dual-Luciferase Reporter Assay System (Promega, Madison, WI) according to the supplier's instructions. Caspase inhibitor zLEHD-fmk was obtained from MBL International (Woburn, MA), and used at a concentration of $5 \mu \mathrm{M}$. Recombinant rat cytokines were purchased from BioLegend (San Diego, CA), eBioscience (San Diego, CA), and Shenandoah Biotechnology (Warwick, PA).

Cell culture. Human intestinal epithelial SK-CO15 and SW480 cells were grown in Dulbecco's modified Eagle's medium supplemented with $10 \%$ fetal calf serum and $1 \%$ antibiotics. IEC- 6 rat cells were grown in Dulbecco's modified Eagle's medium with 5\% fetal calf serum, $2 \mathrm{~mm}$ glutamine, and $0.1 \mathrm{U} \mathrm{ml}^{-1}$ insulin. Cells were maintained in a humidified incubator with $5 \% \mathrm{CO}_{2}$. For long-term cytokine treatment studies, media and cytokines were replenished on days 3 and 5. Scratch-wound assays were performed as described previously. ${ }^{32}$ Briefly, cells were plated on glass coverslips, grown to confluence, and serum-starved shortly before the experiment. Cells then received a single linear wound using a plastic pipette tip attached to a vacuum flask. For live cell imaging experiments, cells were plated on collagen-coated dishes, switched to Optimem with $1 \%$ fetal bovine serum and CK2 inhibitors, scratchwounded after $15 \mathrm{~min}$, and allowed to equilibrate for $10 \mathrm{~min}$ before imaging. Movies were recorded on an Axiovert 200M inverted microscope (Zeiss, Thornwood, NY) fitted with a heated chamber (Brook Industries, Lake Villa, IL). Images were taken every $10 \mathrm{~s}$ for $1 \mathrm{~h}$.

Lentiviral overexpression of CK2 $\alpha$. Full-length rat CK2 $\alpha$ (Open Biosystems, Huntsville, AL) was cloned into a custom lentiviral vector containing a SNAP tag at the N terminus (pLEX-MCS-SNAP). SNAPCK2 $\alpha$ and SNAP control viruses were generated using the Translentiviral packaging kit (Open Biosystems) according to the manufacturer's instructions, and concentrated by centrifugation at $50,000 \mathrm{~g}$ for $90 \mathrm{~min}$ at $4{ }^{\circ} \mathrm{C} .{ }^{33}$ IEC- 6 cells were infected at a multiplicity of infection of 2 , and maintained under puromycin selection $\left(2 \mu \mathrm{g} \mathrm{ml}^{-1}\right)$.

Immunoblotting. Tissue samples and cells in RIPA buffer were sonicated and subjected to bicinchoninic acid analysis. Equal amounts of protein were boiled in Laemmli buffer containing $50 \mathrm{~mm}$ dithiothreitol, and separated by SDS polyacrylamide gel electrophoresis. Proteins were transferred onto nitrocellulose membranes, and blocked with $5 \% \mathrm{wt} / \mathrm{vol}$ dry milk in Tris-buffered saline (TBS) with $0.1 \% \mathrm{vol} / \mathrm{vol}$ Tween-20 (TBST). Next, membranes were incubated overnight with primary antibodies in blocking buffer or $5 \% \mathrm{wt} / \mathrm{vol}$ bovine serum albumin in TBST, and then with horseradish peroxidase-linked secondary antibodies. Horseradish peroxidase was detected using HyGLO reagent (Denville Scientific, Metuchen, NJ).

Confocal microscopy. Frozen tissue sections of $7 \mu \mathrm{m}$ thickness were fixed/permeabilized with $3.7 \% \mathrm{wt} / \mathrm{vol}$ paraformaldehyde in TBS followed by $1 \% \mathrm{vol} / \mathrm{vol}$ Triton X-100 in TBS, or ice-cold ethanol. Slides were then blocked with $2 \% \mathrm{wt} / \mathrm{vol}$ bovine serum albumin in TBS, and incubated with primary antibodies in blocking buffer. Antigen/antibody complexes were visualized using AlexaFluor-labeled secondary antibodies (Invitrogen). Sections were then counterstained with TOPRO-3 iodide, and mounted in $p$-phenylene. Confocal microscopy images were acquired on an LSM510 laser scanning microscope (Zeiss), with software supplied by the vendor. Images for quantitative analyses were taken at identical detector settings.

Immunohistochemistry. Deparaffinized slides were cleared with xylenes, quenched with $0.9 \% \mathrm{vol} / \mathrm{vol} \mathrm{H}_{2} \mathrm{O}_{2}$ in methanol, rehydrated, and boiled in $10 \mathrm{mmoll}^{-1}$ sodium citrate buffer, $\mathrm{pH} 6.0$, for $10 \mathrm{~min}$ at $125^{\circ} \mathrm{C}$. Sections were then blocked in $2 \%$ dry milk in phosphate-buffered saline with $0.1 \%$ Tween-20, treated with an avidin/biotin blocking kit (Vector Laboratories, Burlingame, CA), and incubated with primary antibody in blocking buffer. Antigen/antibody complexes were detected using a $\mathrm{LSAB}+$ System horseradish peroxidase Kit and chromogenic DAB substrate (Dako, Carpinteria, CA). Slides were briefly counterstained with hematoxylin and analyzed on an Axiovert 200M inverted microscope equipped with a $10 \times$ dry objective and AxioCam Rc5 camera (Carl Zeiss), using software supplied by the vendor.

Real-time PCR. RNA was extracted from Isol-RNA embedded tissues by phenol-chloroform extraction using standard protocols. RNA was then purified using the RNeasy Mini Kit (Qiagen, Germantown, MD), and analyzed on a Nanodrop spectrophotometer (Thermo Scientific, Waltham, MA). Real-time reverse-transcriptase-PCR was performed on an iCycler/iQ5 system (Bio-Rad, Hercules, CA), with primers from RealTimePrimers.com (sequences can be found in Supplementary Table S1 online). Results were normalized to Gapdh.

Statistical analyses. Densitometric analyses and fluorescence intensity measurements were performed using NIH ImageJ software (Bethesda, $\mathrm{MD})$. CK2 $\alpha$ nuclear staining in human tissues was assessed in $>50$ cells within $200 \mu \mathrm{m}$ of the crypt bottom, from 10 or more correctly aligned crypts per sample. For CK $2 \alpha$ nuclear/cytoplasmic ratio in IEC- 6 cells, representative areas in the nucleus and cytoplasm of the same cell were analyzed for $\geqslant 100$ cells per experiment and data point. Kymography was performed using the Kymograph (time space plot) Plugin for ImageJ (J. Rietdorf, FMI, Basel, Switzerland, and A. Seitz, EMBL, Heidelberg, Germany). A total of 20 kymographs were generated per condition. Data were analyzed by two-tailed Student's $t$-test, or Dunnett's post hoc test following one-way analysis of variance, using Microsoft Excel or GraphPad Prism 5 software (La Jolla, CA). Results are displayed as mean + s.e.m., or as box-and-whisker plot with mean and quartiles \pm maximum $/ \mathrm{minimum}$. $P<0.05$ was considered statistically significant.

SUPPLEMENTARY MATERIAL is linked to the online version of the paper at http://www.nature.com/mi

\section{ACKNOWLEDGMENTS}

We thank Caroline Addis and Oskar Laur (Emory cloning core facility) for technical assistance. This study was supported by grants from the National Institutes of Health (R01 DK072564, R01 DK061379, and R01 
DK079392 to C.A.P.; R01 DK055679 and DK059888 to A.N.; morphology and tissue culture support from digestive diseases minicenter grant (DDRDC) R24 DK064399); and the Crohn's and Colitis Foundation of America (Research Fellowship Award to S.K. and C.T.C.).

\section{DISCLOSURE}

The authors declared no conflict of interest.

(C) 2013 Society for Mucosal Immunology

\section{REFERENCES}

1. Koch, S. \& Nusrat, A. The life and death of epithelia during inflammation: lessons learned from the gut. Annu. Rev. Pathol. 7, 35-60 (2012).

2. Nava, P. et al. Interferon-gamma regulates intestinal epithelial homeostasis through converging beta-catenin signaling pathways. Immunity 32 , 392-402 (2010).

3. Litchfield, D.W. Protein kinase CK2: structure, regulation and role in cellular decisions of life and death. Biochem. J. 369 (Pt 1), 1-15 (2003).

4. Singh, N.N. \& Ramij, D.P. Protein kinase CK2, an important regulator of the inflammatory response? J. Mol. Med. 86, 887-897 (2008).

5. Dominguez, I., Sonenshein, G.E. \& Seldin, D.C. Protein kinase CK2 in health and disease: CK2 and its role in Wnt and NF-kappaB signaling: linking development and cancer. Cell. Mol. Life Sci. 66, 1850-1857 (2009).

6. McDonnell, M.A. et al. Phosphorylation of murine caspase-9 by the protein kinase casein kinase 2 regulates its cleavage by caspase- 8 . J. Biol. Chem. 283, 20149-20158 (2008).

7. Duncan, J.S. et al. A Peptide-based target screen implicates the protein kinase CK2 in the global regulation of caspase signaling. Sci. Signal. 4, ra30 (2011).

8. Trembley, J.H. et al. Emergence of protein kinase CK2 as a key target in cancer therapy. Biofactors 36, 187-195 (2010).

9. Lin, K.Y. et al. Overexpression of nuclear protein kinase CK2 alpha catalytic subunit (CK2alpha) as a poor prognosticator in human colorectal cancer. PLoS One 6, e17193 (2011).

10. Zou, J., Luo, H., Zeng, Q., Dong, Z., Wu, D. \& Liu, L. Protein kinase CK2alpha is overexpressed in colorectal cancer and modulates cell proliferation and invasion via regulating EMT-related genes. J. Transl. Med. 9, 97 (2011).

11. Yamada, M. et al. Inhibition of protein kinase CK2 prevents the progression of glomerulonephritis. Proc. Natl. Acad. Sci. USA 102, 7736-7741 (2005).

12. Yu, S., Wang, H., Davis, A. \& Ahmed, K. Consequences of CK2 signaling to the nuclear matrix. Mol. Cell. Biochem. 227, 67-71 (2001).

13. Miyata, Y. \& Nishida, E. Evaluating CK2 activity with the antibody specific for the CK2-phosphorylated form of a kinase-targeting cochaperone Cdc37. Mol. Cell. Biochem. 316, 127-134 (2008).

14. Pyerin, W. \& Ackermann, K. Transcriptional coordination of the genes encoding catalytic (CK2alpha) and regulatory (CK2beta) subunits of human protein kinase CK2. Mol. Cell. Biochem. 227, 45-57 (2001).

15. Bauer, C. et al. Colitis induced in mice with dextran sulfate sodium (DSS) is mediated by the NLRP3 inflammasome. Gut 59, 1192-1199 (2010).

16. Sarno, S. et al. Selectivity of 4,5,6,7-tetrabromobenzotriazole, an ATP site-directed inhibitor of protein kinase CK2 ('casein kinase-2'). FEBS Lett 496, 44-48 (2001).
17. Ponce, D.P. et al. Phosphorylation of AKT/PKB by CK2 is necessary for the AKT-dependent up-regulation of beta-catenin transcriptional activity. J. Cell. Physiol. 226, 1953-1959 (2011).

18. Tawfic, S., Yu, S., Wang, H., Faust, R., Davis, A. \& Ahmed, K. Protein kinase CK2 signal in neoplasia. Histol. Histopathol. 16, 573-582 (2001).

19. Krehan, A., Ansuini, H., Bocher, O., Grein, S., Wirkner, U. \& Pyerin, W. Transcription factors ets1, NF-kappa B, and Sp1 are major determinants of the promoter activity of the human protein kinase CK2alpha gene. J. Biol. Chem. 275, 18327-18336 (2000).

20. Parhar, K., Morse, J. \& Salh, B. The role of protein kinase CK2 in intestinal epithelial cell inflammatory signaling. Int. J. Colorectal Dis. 22, 601-609 (2007).

21. Mead, J.R., Hughes, T.R., Irvine, S.A., Singh, N.N. \& Ramji, D.P. Interferon-gamma stimulates the expression of the inducible cAMP early repressor in macrophages through the activation of casein kinase 2. A potentially novel pathway for interferon-gamma-mediated inhibition of gene transcription. J. Biol. Chem. 278, 17741-17751 (2003).

22. Su, Y.W., Xie, T.X., Sano, D. \& Myers, J.N. IL-6 stabilizes twist and enhances tumor cell motility in head and neck cancer cells through activation of casein kinase 2. PLoS One 6, e19412 (2011).

23. Krehan, A. et al. Ets 1 is a common element in directing transcription of the alpha and beta genes of human protein kinase CK2. Eur. J. Biochem. 268, 3243-3252 (2001).

24. Ackermann, K., Neidhart, T., Gerber, J., Waxmann, A. \& Pyerin, W. The catalytic subunit alpha' gene of human protein kinase CK2 (CSNK2A2): genomic organization, promoter identification and determination of Ets1 as a key regulator. Mol. Cell. Biochem. 274, 91-101 (2005).

25. Seno, H., Miyoshi, H., Brown, S.L., Geske, M.J., Colonna, M. \& Stappenbeck, T.S. Efficient colonic mucosal wound repair requires Trem2 signaling. Proc. Natl. Acad. Sci. USA 106, 256-261 (2009).

26. Edelblum, K.L., Yan, F., Yamaoka, T. \& Polk, D.B. Regulation of apoptosis during homeostasis and disease in the intestinal epithelium. Inflamm. Bowel Dis. 12, 413-424 (2006).

27. Raleigh, D.R. et al. Occludin S408 phosphorylation regulates tight junction protein interactions and barrier function. J. Cell Biol. 193, 565-582 (2011).

28. Tapia, J.C., Torres, V.A., Rodriguez, D.A., Leyton, L. \& Quest, A.F. Casein kinase 2 (CK2) increases survivin expression via enhanced beta-catenin-T cell factor/lymphoid enhancer binding factor-dependent transcription. Proc. Natl. Acad. Sci. USA 103, 15079-15084 (2006).

29. Barrett, R.M., Colnaghi, R. \& Wheatley, S.P. Threonine 48 in the BIR domain of survivin is critical to its mitotic and anti-apoptotic activities and can be phosphorylated by CK2 in vitro. Cell Cycle 10, 538-548 (2011).

30. Koch, S. et al. The Wnt antagonist Dkk1 regulates intestinal epithelial homeostasis and wound repair. Gastroenterology 141, 259-268 (2011), 268 e251-258.

31. Grassl, G.A., Valdez, Y., Bergstrom, K.S., Vallance, B.A. \& Finlay, B.B. Chronic enteric salmonella infection in mice leads to severe and persistent intestinal fibrosis. Gastroenterology 134, 768-780 (2008).

32. Koch, S. et al. Dkk-1 inhibits intestinal epithelial cell migration by attenuating directional polarization of leading edge cells. Mol. Biol. Cell. 20, 4816-4825 (2009).

33. Burns, J.C., Friedmann, T., Driever, W., Burrascano, M. \& Yee, J.K. Vesicular stomatitis virus $\mathrm{G}$ glycoprotein pseudotyped retroviral vectors: concentration to very high titer and efficient gene transfer into mammalian and nonmammalian cells. Proc. Natl. Acad. Sci. USA 90, 8033-8037 (1993). 